\title{
Compliance in Celiac Disease Patients
}

\author{
Parveen Malhotra*, Vani Malhotra, Smiti Nanda, Vandana Rani, Meenakshi Chauhan and Isha Pahuja \\ Department of Medical Gastroenterology, Obstetrics and Gynecology, India
}

Submission: September 06, 2019; Published: November 05, 2019

*Corresponding author: Dr. Parveen Malhotra, Senior Professor \& Head, Department of Medical Gastroenterology, PGIMS, Rohtak, India

\begin{abstract}
Background: Celiac disease (CD), is a chronic immune-mediated disorder of small intestine that occurs in genetically predisposed populations due to permanent intolerance to wheat gliadins and other cereal prolamins. The epidemiology of CD has iceberg characteristics with more undiagnosed cases. The diagnosis of $\mathrm{CD}$ is currently based on both typical small bowel biopsy findings with clinical and serological parameters.
\end{abstract}

Aims: The aim of study was to determine compliance regarding gluten restricted diet in confirmed cases of celiac disease.

Materials and Methods: Two hundred and twenty-five confirmed cases of celiac disease on basis of clinical history, physical examination, raised serum IgAttg level and duodenal biopsies were followed regularly in celiac clinic and telephonically for one year and then compliance rate regarding strict gluten in diet was calculated.

Results and Conclusions: The compliance rate regarding strict gluten restricted diet was Ninety (89.77\%). It was more in females and in older age group in comparison to males and younger age group.

Keywords: Celiac disease; Duodenal biopsy; Gluten; Compliance; Small intestine; Transglutaminase; Endomysium; Wheat; Rye; Oat; Barley; Proline content; Clinical gastrointestinal; Intraepithelial lymphocytes; Crypt hyperplasia

Abbreviations: CD: Celiac Disease; GI: Gastrointestinal; IHC: Immunohistochemical; IELs: Intraepithelial Lymphocytes

\section{Introduction}

Celiac disease (CD) is a chronic immune-mediated disorder of small intestine that occurs in genetically predisposed populations [1]. The pathogenesis entails a $\mathrm{T}$ cell mediated immune response with production of auto antibodies directed against tissue transglutaminase or endomysium. Wheat, rye, oat and barley prolamins are toxic for celiac patients due to their high glutamine and proline content [2]. Initially the suspicion of CD was based on clinical gastrointestinal (GI) symptoms. Subsequently, the disease has been found with variety of atypical symptoms and even in asymptomatic subjects [3]. Celiac disease affects people in all parts of the world. The histologic changes in CD vary from severe villous atrophy to more subtle changes (with or without increased density of intraepithelial lymphocytes and crypt hyperplasia). Although villous atrophy is not specific to CD. Serology has become increasingly relevant to CD diagnosis. Anti-tissue transglutaminase antibodies are the most sensitive test for CD [2].

\section{Material and Methods}

Two hundred and twenty-five confirmed cases of celiac disease on basis of clinical history, physical examination, raised serum IgAttg level and duodenal biopsies were followed regularly in celiac clinic and telephonically also for one year and then compliance rate regarding strict gluten restriction in diet was calculated. The biopsy was taken from second part of duodenum through eosophagoduodenoscopy with the assessment of duodenal endoscopic markers including scalloping of folds, grooving, and nodularity of mucosa. Histopathological diagnosis was established on routine hematoxylin and eosin stained sections [4]. Immunohistochemical (IHC) staining [5] with antihuman CD3 antibody was done for evaluating intraepithelial lymphocytes (IELs) with positive (tonsillar tissue) and negative (substituting the primary antibody with an antibody of irrelevant specificity) controls. The histopathological grading was performed as per modified Marsh grading [6]. 


\section{Discussion}

Celiac Disease is also known as intestinal infantilism, idiopathic steatorrhea, non-tropical sprue, and gluten sensitive enteropathy. Various etiological factors are considered for CD including genetic (HLA class II antigen) and environmental risk factors [7] including GI infections [8]. Gliadin is a glycoprotein extract from gluten, directly toxic to the enterocytes of individuals with CD. Transglutaminase enzyme crosslinks gliadin and causes specific deamination of glutamine into glutamic acid. With such deamination, the gliadin peptides are able to be more efficiently presented to gliadin-reactive CD-4 T cells.

Without Transglutaminase, it is believed that gliadin is less immunogenic. Thus, Transglutaminase auto antibodies play a role in disease pathogenesis, but lacks sufficient supportive evidence [9]. The epidemiology of CD has iceberg characteristics as there are more undiagnosed cases. The female-to male ratio is $2: 1$. The prevalence of $\mathrm{CD}$ is globally $1 \%$. The prevalence of $\mathrm{CD}$ in India is nearly the same as that in Western Caucasian populations [10]. Adult celiac were more than children in our study. In both groups, females were predominant. Maximum number of patients presented with GI symptoms than atypical symptoms in which diarrhea was the most common complaint as seen in other studies in literature [11-16].

In our study we found compliance rate to be $89.77 \%$ i.e. in 202 patients in total group of 225 patients, in comparison to JC Chauhan et al, [17] who reported compliance rate of $75 \%$. They found decreased dietary compliance during 10-17 yr of age ( $44 \%$, compared to $>80 \%$ in $<9 y$ r age group) but in contrast in our study we found compliance to be more in adults then younger age group. Ljungman et al, [18] reported compliance rates of $93 \%$ at 12 years of age decreasing to $76 \%$ in $15-17$ yrs age group. In the present study, non-compliance was more common in female patients i.e., 60\%. Higher dietary noncompliance in female patients may be explained by socio-cultural factors, family pressure and gender bias. Results of study by Anson et al, [19] also show that maternal education is important factor affecting compliance, which may be because mother is responsible for preparation of food items and providing GFD and same fact was found to be totally correct in our study group also. In our study we concluded that non-compliance was higher in Joint families and with more numbers of siblings. When dietary compliant and dietary non-compliant patients were compared, it was found $90 \%$ of dietary compliant patients presented with typical symptoms and $10 \%$ presented with atypical symptoms because patients presenting with typical symptoms had visible improvement in their symptoms. Fabiani et al. [20] also reported higher compliance in symptomatic patients as compared to asymptomatic screen detected patients. Parents of the dietary compliant groups had better understanding of Celiac disease than those of non-compliant group. Butterworth et al have reported lower compliance in south Asian migrants to UK, who were less satisfied with the explanation by the physician [21].

In our study higher compliance rate can be attributed to greater effort on counseling of patients and family members and highlighting repeatedly the importance of gluten restricted diet on follow up and even telephonically also. Anson et al [19] study also showed similar correlation of parental knowledge and dietary compliance [1]. Thus, results of present study show that compliance is higher when parents have better knowledge about celiac disease, understand value of gluten free diet and are able to handle the menu better. These results highlight importance of proper counseling and reinforcement during subsequent visits. Difficulty in maintaining dietary compliance at school and at family party/marriage, was higher in dietary non-compliant group compared to the dietary compliant. Gluten containing food as main dietary item, served at above places, was a problem for both dietary compliant and non-compliant group. Present study findings show that dietary non-compliance is more common when preparing gluten free food items is considered a financial burden. Similar finding also has been reported from Anson et al [19]. As more and more patients are being diagnosed from lower socio-economic status, counseling regarding cheap food items and easily prepared GF food items will probably help this group (Figure $1 \& 2$ ).

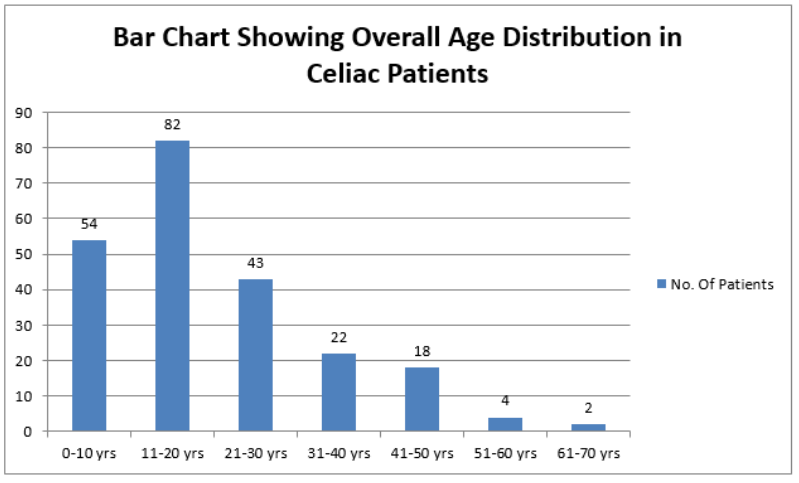

Figure 1: Bar Chart Showing Overall Age Distribution in Celiac Patients. 


\section{Bar Chart Showing Age Along With Sex Distribution in Celiac Patients}

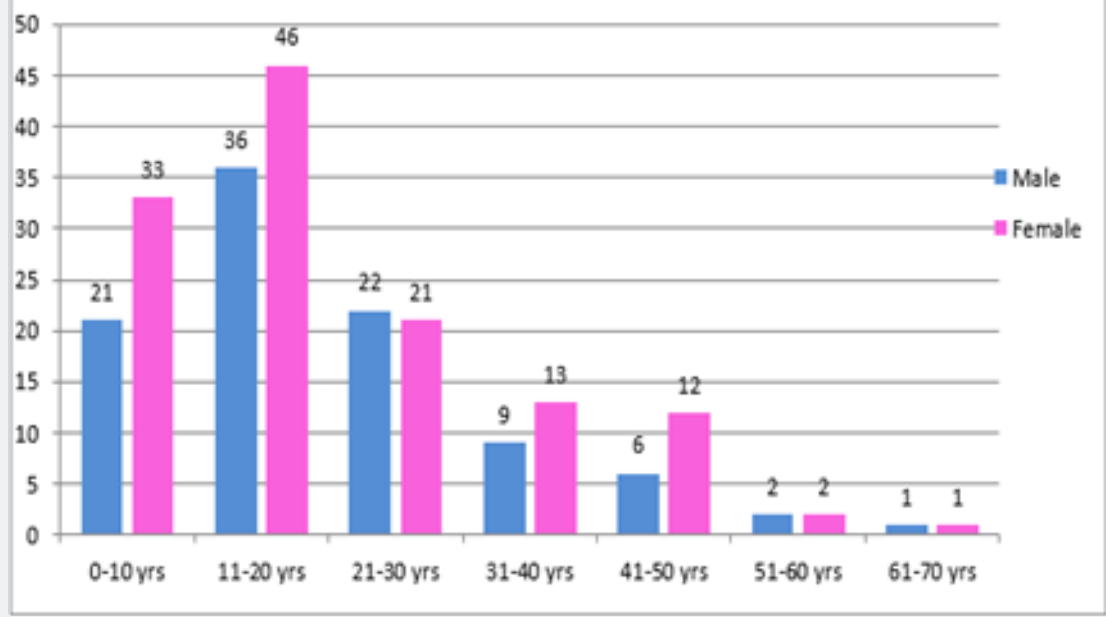

Figure 2: Bar Chart Showing Age Along with Sex Distribution in Celiac Patients.

\section{Conclusion}

The first and foremost important point in celiac disease is to make patient and family members understand the importance of life-long restriction of gluten in diet. For this onus lies on shoulders of treating Gastroenterologist/Physician which requires proper counseling at initial diagnosis and on regular follow up of patients. This will lead to increasing compliance in celiac patients in whom the only treatment available at this point is gluten restriction in diet. For increasing the compliance among patients, there is also requirement of training of medical professionals and alternative medicine practitioners, for not restarting gluten in diet under cover of steroids because they temporarily mask the development of symptoms due to re-entry of gluten in body but damage of gluten on intestine goes unabated and even side effects of immunosuppressant's like hypertension, diabetes mellitus, osteoporosis etc. develop. Other important issue for increasing compliance is to recognize hidden source of gluten being taken in the diet, of which patient is unaware, thus there is unsatisfactory clinical, biochemical and histological recovery. The reasons for it are adulteration of gluten in diet due to common kitchen practices for preparing food for whole of family, using same utensils or consumption of market products thinking to be gluten free but in reality containing minute amounts of gluten in diet and even frequent and heavy use of lip-stick, as it contains gluten as a base. In school going children, the reason came out to be sharing of food with other children.

\section{References}

1. Harris LA, Park JY, Voltaggio L, Himlin DL (2012) Celiac disease clinical, endoscopic and histopathologic review. Gastrointest endosc 76(3): 625-640
2. Volta U, Villanacci V (2011) Celiac disease: diagnostic criteria in progress. Cell Mol Immunol 8(2): 96-102.

3. Serra S, Jani PA (2006) An approach to duodenal biopsies. J Clin Pathol 59(11): 1133-1150.

4. Lohi S, Mustalahti K, Kaukinen K, Laurila K, Collin P, et al. (2007) Increasing prevalence of celiac disease over time. Aliment Pharmacol Ther 26(9): 1217-1225.

5. Murray AJ (1999) The widening spectrum of celiac disease. Am J Clin Nutr 69: 354-365.

6. Dowd B, Walker-Smith J (1974) Samuel Gee, Aretaeus and the celiac affection. Br Med J 2(5909): 45-47.

7. Akobeng AK, Ramanan AV, Buchan I, Heller RF (2006) Effect of breast feeding on risk of celiac disease: a systematic review and meta-analysis of observational studies. Arch Dis Child 91(1): 39-43.

8. Myleus A, Ivarsson A, Webb C, Danielsson L, Hernell O, et al. (2009) Celiac disease revealed in $3 \%$ of Swedish 12 -year-olds born during an epidemic. J Pediatr Gastroenterol Nutr 49(2): 170-176.

9. Guandalini S (2007) The influence of gluten: weaning recommendations for healthy children and children at risk for celiac disease. Nestle Nutr Workshop Ser Pediatr Program 60: 139-151

10. Plot L, Amital H (2009) Infectious associations of celiac disease. Autoimmun Rev 8(4): 316-319.

11. Van de Kamer J, Weijers H, Dicke W (1953) Celiac disease- Some experiments on the cause of the harmful effect of wheat gliadin. Acta Paediatr Scand 44(5): 223-231.

12. Walker MM, Murray JA (2011) An update in the diagnosis of celiac disease. Histopathology 59(2): 166-179.

13. Gujral N, Freeman HJ, Thomson AB (2012) Celiac disease: Prevalence, diagnosis, pathogenesis and treatment. World J Gastroenterol 18(42): 6036-6059.

14. Collin P, Reunala T, Pukkala E, Laippala P, Keyrilainen O, et al. (1994) Celiac disease-associated disorders and survival. Gut 35(9): 12151218 
15. Lauret E, Rodrigo L (2013) Celiac disease and autoimmune associated conditions. Biomed Res Int 2013: 127589.

16. Kingham J, Parker D (1998) The association between primary biliary cirrhosis and celiac disease: a study of relative prevalence. Gut 42(1): 120-122.

17. JC Chauhan, P Kumar, AK Dutta, S Basu, A Kumar (2010) Assessment of Dietary Compliance to Gluten Free Diet and Psychosocial Problems in Indian Children with Celiac Disease. Indian J Pediatr 77(6): 649-654.

18. Ljungman G, Myrdal U (1993) Compliance in teenagers with celiac disease: a Swedish follow-up study. Acta Paediatr 82(3): 235-238.
19. Anson O, Weizman Z, Zeevi Naomi (1990) Celiac disease: parental knowledge and attitudes of dietary compliance. Pediatric 85(1): 98103.

20. Fabiani E, Catassi C, Villari A, Gismondi P, Pierdomenico R, et al. (1996) Dietary compliance in screening-detected celiac disease adolescents. Acta Paediatr 412(Suppl): 65-67.

21. Butterworth J R, Banfield L M, Iqbal T H, Cooper B T (2004) Factors relating to compliance with a gluten-free diet in patients with celiac disease: comparison of white Caucasian and South Asian patients. Clin Nutri 23(5): 1127-1134.

This work is licensed under Creative Commons Attribution 4.0 License DOI: 10.19080/ARGH.2019.14.555879 